\title{
Structural analysis of fibrillar polymorphs in AD (Alzheimer Disease) brain tissue and Pair distribution function of associated fibrils \\ Prakash Nepal ${ }^{1}$, Abdullah Al Bashit ${ }^{2}$, Lee Makowski ${ }^{3}$ \\ ${ }^{1}$ Dept. of Bioengineering ${ }^{2}$ Northeastern University, ${ }^{3}$ Northeastern University \\ p.nepal@northeastern.edu
}

X-ray microdiffraction in the SAXS (Small Angle X-ray scattering) and WAXS (Wide Angle X-ray scattering) regimes can be used to probe the structural organization of amyloid and NFT (Neurofibrillary tangle) deposits in thin section of tissue from AD subjects. The Wide-angle X-ray scattering from these deposits is dominated by a layer line at 4.7 AX-ray microdiffraction in the SAXS (Small Angle X-ray scattering) and WAXS (Wide Angle Xray scattering) regimes can be used to probe the structural organization of amyloid and NFT (Neurofibrillary tangle) deposits in thin section of tissue from $\mathrm{AD}$ subjects. The Wide-angle X-ray scattering from these deposits is dominated by a layer line at $4.7 \AA$ spacing which provides insight into fibrillar structure. The polymorphism of these structures is being analyzed using data from the SAXS regime that generates information about cross-sectional shape/ size and cylindrical pair distribution function of fibrils involved.

The cylindrical pair distribution function differs from the conventional pair distribution function derived from solution scattering in that it corresponds to a histogram of interatomic distances projected on a plane perpendicular to fiber axis. In this study, the cylindrical pair distribution function is being utilized to determine the maximum diameters of fibrils and fibrillar aggregates within intact tissue. This work is resulting in mapping of structural variations of pathological protein deposits within brain tissue which will contribute to a better understanding of the molecular basis of the progression of AD.

This work is supported by NIH Grant number R21AG068972. 